\title{
A Mathematical Simulation of Growth of Fusarium in Maize Ears After Artificial Inoculation
}

\author{
D. W. Stewart, L. M. Reid, R. W. Nicol, and A. W. Schaafsma
}

First and second authors: Eastern Cereal and Oilseed Research Centre, Central Experimental Farm, Agriculture and Agri-Food Canada, Ottawa, Ontario, Canada K1A 0C6; third author: University of Western Ontario, Department of Plant Science, London, Ontario, N6A 5B8; and fourth author: Ridgetown College, University of Guelph, Ridgetown, Ontario, N0P 2C0.

Accepted for publication 24 January 2002.

\begin{abstract}
Stewart, D. W., Reid, L. M., Nicol, R. W., and Schaafsma, A. W. 2002. A mathematical simulation of growth of Fusarium in maize ears after artificial inoculation. Phytopathology 92:534-541.

Fusarium spp. in maize can contaminate the grain with mycotoxins if environmental conditions are favorable for fungal growth. To quantify the relationship between growth of Fusarium spp. and environmental conditions, a mathematical model was developed to simulate growth of $F$. graminearum and $F$. verticillioides on maize ears following silk inoculation in field experiments from 1992 to 1995 . Each species was inoculated separately and as a mixture of the two for 3 of the 4 years on one maize hybrid. Disease progress in ears was measured by a visual rating scale that was converted to percent visual infection. Measurements were made at regular time intervals after silks were inoculated 5 days after silk emergence. Differential equations were used to relate growth rates of Fusarium spp. in maize ears to hourly air temperature and relative hu-

midity and to daily precipitation. Integration of these equations over time produced quantitative estimates of fungal growth. Model calculations compared well with measurements $\left(R^{2}=0.931\right.$, standard error of estimate [SEE $]=2.11 \%)$ of percent visual disease infection of maize ears over 3 years. The model was tested against a second set of data $\left(R^{2}=0.89\right.$, SEE $=5.9 \%)$ in which silks were inoculated at nine different times after first silk emergence for each of 2 years (1994 and 1995) with the two species of fungi on the same maize hybrid. At this time, a silk function was developed to account for changes in the susceptibility of silks to disease. F. graminearum responded to wet conditions more than F. verticillioides, and for the conditions of this experiment, grew much faster than $F$. verticillioides when inoculated separately. When they were inoculated together, $F$. graminearum growth rates were much lower, indicating some interference by $F$. verticillioides. During 1993, weather conditions before inoculation reduced the growth of both species in silks.
\end{abstract}

Growth of Fusarium in ears of maize (corn, Zea mays L.) is an important concern for maize production due to a negative effect on yield and grain quality. These fungal species produce mycotoxins that can adversely affect growth rates of livestock fed contaminated maize $(8,11)$. In Canada, the toxins of most concern are deoxynivalenol (DON, vomitoxin) and zearalenone, produced by $F$. graminearum Schwabe [teleomorph $=$ Gibberella zeae (Schwein.) Petch] followed by fumonisin $\mathrm{B}_{1}$, produced by $F$. verticillioides (Sacc.) Nirenberg $[=F$. moniliforme, teleomorph $=$ G. fujikuroi (Sawada) Wr.] (21). These fungi can infect ears through wounds caused by birds and insects or from colonization of silks and growth through the silk channels to the kernels $(19,20)$. In years of severe infection by $F$. graminearum, the most common route is through the silks (21).

Year-to-year variation in amounts of disease is highly dependent on weather conditions although the response to weather varies among species. For example, $F$. graminearum grows better in wet years than $F$. verticilliodes $(8,20)$. Quantification of weather effects on growth of Fusarium, measured as disease development by linear regression analysis, was done by Moschini and Fortugno (9) for wheat and Vigier et al. (21) for maize. A mechanistic model of mold growth of Aspergillus flavus Link ex Fries mold growth based on differential equations was developed by Pitt (10). However, this model simulated fungal growth only under controlled environmental conditions. Various types of simulation

Corresponding author: D. W. Stewart; E-mail address: stewartdw@em.agr.ca

Publication no. P-2002-0320-01R

(c) 2002 The American Phytopathological Society models of diseases for field conditions have been developed. Simulators such as EPIMAY (22) for southern corn leaf blight were cited by Campbell and Madden (4). To our knowledge, there has been no such model for Fusarium spp. on maize.

The objective of this study was to develop a mechanistic or conceptual model of Fusarium growth in maize ears under field conditions after artificial inoculation with two species of Fusarium ( $F$. graminearum and $F$. verticillioides), separately and together, so that the amounts of Fusarium percent visual infection, alone and in competition, could be estimated from weather parameters. This is a first step in building a general model for calculating levels of toxins over regions based on weather parameters.

\section{THEORY AND APPROACHES}

Model description. Many processes can best be described by how they change with time using a differential equation. In this study, the model was based on a differential equation relating rate of growth of a single species to amount of growth $(G)$, a temperature function $\left(T_{F}\right)$, and an ear wetness function $\left(W_{F}\right)$. The potential for exponential growth was made possible by making the rate of growth directly proportional to the amount of growth. That is

$$
d G / d t=a_{1} G T_{F} W_{F}
$$

The amount of growth $(G)$ is determined by visual inspection of maize ears and is expressed as the percentage of the ear that is visually infected by the fungi being studied. Therefore, $G$ will be referred to as percent visual infection. More detail on how $G$ was 
measured is provided below. The temperature function was an Arrhenius-like equation from Pitt (10):

$$
T_{F}=A \exp \left[-\left(\frac{\alpha^{2}}{\left(T-T_{\min }\right)}+\frac{\beta^{2}}{\left(T-T_{\max }\right)}\right)\right] \quad T_{\min }<T<T_{\max }
$$

where $T$ is hourly temperature (either silk or air, degree Celsius), $T_{\min }$ is a minimum temperature (degree Celsius) for growth, $T_{\max }$ is a maximum temperature (degree Celsius) for growth, $\alpha$ and $\beta$ are shape factors (degree Celsius to the one-half $\left[{ }^{\circ} \mathrm{C}^{1 / 2}\right]$ ), and $A$ is a scaling factor (dimensionless). All shape factors and other coefficients are listed with units in Table $1 . T_{F}$ is set to zero for temperatures less than $T_{\min }$ and greater than $T_{\max }$. An optimum temperature was defined by setting the differential of equation 2 to zero when $T$ was equal to the optimum temperature $\left(T_{\text {opt }}\right)(10)$. By rearranging, $\beta$ is expressed as

$$
\beta=\alpha\left(T_{\max }-T_{\mathrm{opt}}\right) /\left(T_{\mathrm{opt}}-T_{\min }\right)
$$

The scaling factor $(A)$ was calculated by setting $T_{F}$ equal to 1.0 when $T$ was equal to $T_{\text {opt }}$ for $F$. graminearum and $T_{F}$ was equal to 1.08 for $F$. verticillioides. The slightly higher value of 1.08 corresponded to a higher growth rate at higher temperatures for $F$. verticillioides as explained below. This left only $\alpha, T_{\max }, T_{\min }$, and $T_{\text {opt }}$ as unknowns, but values of these coefficients will determine the shape of the temperature response function.

Values for these coefficients were difficult to determine. The data on growth rates from Reid et al. (15) indicated values of 28 and $31^{\circ} \mathrm{C}$ for $T_{\mathrm{opt}}$ and $T_{\max }$ for $F$. graminearum, respectively. Values of $T_{\text {opt }}$ and $T_{\max }$ for $F$. verticilloides were set at 31 and $35^{\circ} \mathrm{C}$, respectively, to correspond with evidence that $F$. verticilloides grows faster at higher temperatures (17). The function for $F$. verticilloides was multiplied by a factor of 1.08 so that the two species had similar temperature response functions for temperatures below $28^{\circ} \mathrm{C}$ as indicated by the data from Reid et al. (15). Finally, $\alpha$ and $T_{\min }$ were set at 3 and $15^{\circ} \mathrm{C}$, respectively, to correspond to Alberts et al. (1), who showed considerable growth of $F$. verticilloides at $20^{\circ} \mathrm{C}$, and the temperature response functions of Marin et al. (6), with growth as low as $10^{\circ} \mathrm{C}$ depending on water availability. The temperature response function will be discussed in more detail below.

The ear wetness function was a modification of the model of Rao et al. (12) in which a film of water $(R)$ was assumed to adhere to the surface of the ear. Rao et al. (12) assumed a maximum thickness of $0.8 \mathrm{~mm}$, which we increased to $1.0 \mathrm{~mm}$ to allow for some of the water to occur in the husks and the husk-kernel interface. It is the water status of the kernels not the sheath surface that will influence the spread of the fungi. Precipitation $(P)$ is the rainfall or irrigation in millimeters of water. Changes in the thickness of this water film was expressed mathematically by

$$
\Delta R=-a_{2}\left(V_{p s}-V_{p}\right)+P
$$

where $\Delta R$ is the change in thickness of the film during a time step in the model (in this case $1 \mathrm{~h}$ ), $V_{p}$ is the vapor pressure, and $V_{p s}$ is the saturated vapor pressure. More detail on time steps is given below. The equation $V_{p s}-V_{p}$ is the vapor pressure gradient and was calculated from relative humidity and air temperature. The film thickness was never less than zero or greater than the maximum value of $1 \mathrm{~mm}$. The coefficient $a_{2}$ was a function of the physical dimensions of the ear and the thickness of the earatmosphere boundary layer. The latter was, in turn, related to the wind speed at ear height in the maize canopy (12). In addition, $a_{2}$ was related to the water diffusion properties of the maize ear husk. Because wind speed at the ears was not available in these experiments, is seldom measured in general, and very little is known on water transfer properties of husks, a simple coefficient was assumed that represented average wind conditions and ear characteristics. The vapor pressure deficit was assumed to be the main environmental variable controlling water loss from the ear. The wetness function $\left(W_{F}\right)$ was expressed as

$$
W_{F}=\left(1+a_{3} R\right)
$$

where $a_{3}$ is an empirical coefficient.

The differential equations were solved by using simple trapezoidal integration. For example, equation 1 became

$$
G_{i}=G_{E}+\sum_{i=n}^{N} a_{1} G_{i-1} T_{F} W_{F} \Delta t
$$

That is, we started with an initial small value $G_{E}$ and stepped through the growth period $1 \mathrm{~h}$ at a time. The subscript $i$ represents the number of hours after inoculation, $\Delta t$ is the time step of $1 \mathrm{~h}, n$ is the time when the fungi reached the kernels from the inoculation site in the silks, and $N$ is the total number of hours from inoculation to the end of the growth period. However, in order to determine $G_{E}$ and $n$, the following equation was needed to describe growth of the fungi down the silk channels:

$$
d G / d t=c_{1} a_{5} T_{F} W_{F} S_{F}
$$

where $c_{1}$ is an empirical coefficient, $a_{5}$ is a coefficient to describe the inhibiting effects of weather on silk growth before inoculation, and $S_{F}$ is a silk function to account for silk aging effects as described by Reid et al. (13). The $a_{5}$ coefficient is related to precipitation deficits (precipitation - potential evapotranspiration)

\begin{tabular}{|c|c|c|}
\hline Coefficient & Name & Values (SE) \\
\hline$a_{1}$ & Ear growth & $0.0243(0.00516) \mathrm{h}^{-1}$ \\
\hline$a_{2}$ & Ear air exchange & $\begin{array}{l}0.000305(0.000013) \\
\mathrm{mm} / \mathrm{kPa}\end{array}$ \\
\hline$a_{3}(\mathrm{Gr})$ & Wetness response & $1.710(0.545) \mathrm{mm}^{-1}$ \\
\hline$a_{3}(\mathrm{Ve})$ & Wetness response & $0.930(0.410) \mathrm{mm}^{-1}$ \\
\hline$a_{5} 1993$ & Pre-silk water stress & $0.1918(0.0098)$ \\
\hline$a_{5} 1992$ & Pre-silk water stress & 1.0 \\
\hline$a_{5} 1994$ & Pre-silk water stress & 1.0 \\
\hline$b_{1}$ & First order toxin & $3.66(0.540) \mu \mathrm{g} / \mathrm{g} / \%$ \\
\hline$b_{2}$ & Second order toxin & $0.0253(0.00916) \mu \mathrm{g} / \mathrm{g} / \%^{2}$ \\
\hline$c_{1}$ & Silk growth coefficient & $0.00413 \% / \mathrm{h}$ \\
\hline$c_{2}$ & Silk interference coefficient & $0.500 \% \%^{-1}$ \\
\hline$c_{3}$ & Ear interference coefficient & $0.316 \%^{-1}$ \\
\hline$d_{1}$ & Silk age $y$-intercept & -0.25 \\
\hline$d_{2}$ & Silk age 1 st critical level & 35 MGDD \\
\hline$d_{3}$ & Silk age 2 nd critical level & 55 MGDD \\
\hline$d_{4}$ & Silk age slope & $0.050(0.017) \mathrm{MGDD}^{-1}$ \\
\hline$d_{5}$ & Silk age plateau factor & 0.20 \\
\hline$T_{\max }(\mathrm{Gr})$ & $\begin{array}{l}\text { Maximum temperature for fungal } \\
\text { growth }\end{array}$ & $31^{\circ} \mathrm{C}$ \\
\hline$T_{\max }(\mathrm{Ve})$ & $\begin{array}{l}\text { Maximum temperature for fungal } \\
\text { growth }\end{array}$ & $35^{\circ} \mathrm{C}$ \\
\hline$T_{\mathrm{opt}}(\mathrm{Gr})$ & $\begin{array}{l}\text { Optimum temperature for fungal } \\
\text { growth }\end{array}$ & $28^{\circ} \mathrm{C}$ \\
\hline$T_{\text {opt }}(\mathrm{Ve})$ & $\begin{array}{l}\text { Optimum temperature for fungal } \\
\text { growth }\end{array}$ & $31^{\circ} \mathrm{C}$ \\
\hline$A(\mathrm{Gr})$ & Scale factor (temperature function) & 1.00 \\
\hline$A(\mathrm{Ve})$ & Scale factor (temperature function) & 1.08 \\
\hline$T_{\min }$ & Minimum temperature & $15^{\circ} \mathrm{C}$ \\
\hline$\alpha$ & Shape factor (temperature function) & $3^{\circ} \mathrm{C}^{1 / 2}$ \\
\hline$G_{0}$ & Initial percent infection in silks & $0.3 \%$ \\
\hline$G_{E}$ & Initial percent infection in ears & $0.6 \%$ \\
\hline
\end{tabular}
for a 40-day period before inoculation. Potential evapotranspiration was calculated from maximum and minimum air temperatures using the equations of Baier and Robertson (3) and

TABLE 1. List of coefficients which appear in the derivation of the model in the text with values, standard errors in brackets and unit ${ }^{\mathrm{a}}$

a Coefficients are dimensionless when no units are shown. Gr = Fusarium graminearum, $\mathrm{Ve}=F$. verticilloides . 
Baier (2). The silk function (dimensionless) was assumed to be equal to one for conditions of experiment $\mathrm{A}$ when inoculation took place 5 days after silking. We will return to the silk function when describing how the model was compared with results from experiment B. Solving equation 7 by simple trapezoidal integration resulted in

$$
G_{i}=G_{0}+\sum_{i=1}^{n} c_{1} a_{5} T_{F} W_{F} S_{F} \Delta t
$$

Therefore, during the silk phase, percent infection increased from an initial value of $G_{0}$ to a value of $G_{E}$ in $n$ days. $G_{0}$ will correspond to the amount of inoculum.

In this study, growth $\left(G_{1}\right.$ and $\left.G_{2}\right)$ of two species were studied. Therefore, two sets of equations were used that differed only in the temperature function and the value of the $a_{3}$ (wetness) coefficient. There was also a mixed inoculum that involved interference of one organism with the other. The assumption was made that $F$. verticillioides $\left(G_{2}\right)$ interfered with the growth of $F$. graminearum $\left(G_{1}\right)$ based on the results of Reid et al. (15). Therefore, for the case when inoculum was mixed, equations for the two species were altered as follows. For $F$. graminearum with mixed inoculum, the following two equations replaced equations 6 and 8 , respectively,

$$
G_{1 i}=G_{E}+\sum_{i=n}^{N} a_{1} G_{1(i-1)} T_{F} W_{F}\left(1-c_{2} G_{2(i-1)}\right) \Delta t
$$

and

$$
G_{1 i}=G_{01} / 2+\sum_{1=1}^{n} c_{1} a_{5} T_{F} W_{F} S_{F}\left(1-c_{3} G_{2(i-1)}\right) \Delta t
$$

The corresponding equations for $F$. verticillioides were

$$
G_{2 i}=G_{E}+\sum_{i=n}^{N} a_{1} G_{2(i-1)} T_{F} W_{F} \Delta t
$$

and

$$
G_{2 i}=G_{02} / 2+\sum_{i=1}^{n} c_{1} a_{5} T_{F} W_{F} S_{F} \Delta t
$$

The interference coefficients $\left(c_{2}\right.$ and $\left.c_{3}, \%^{-1}\right)$ in equations 9 and 10 , when multiplied by the percent visual infection of $F$. verticillioides, slowed down the growth of $F$. graminearum. The initial values of growth were divided by two because only one-half the inoculums was used per species in the mixed treatment.

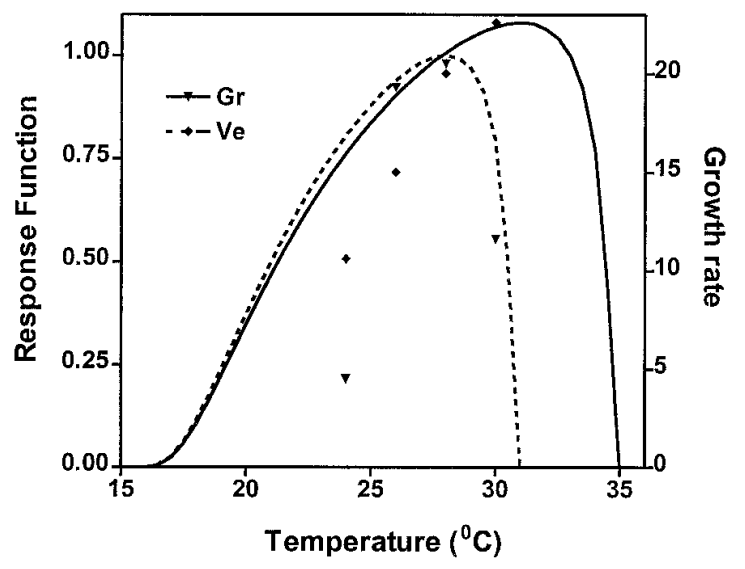

Fig. 1. Temperature function (lines) for Fusarium graminearum (Gr) and $F$. verticillioides ( $\mathrm{Ve})$ as used in the simulation model described in the text compared with growth data (symbols) from Reid et al. (15).
The next stage of the model was to relate growth of Fusarium to the concentration of toxin in an ear $\left(T_{X}\right)$. To do this we used a second order polynomial:

$$
T_{X}=b_{1} G+b_{2} G^{2}
$$

Experimental procedures. Two experiments were used in this study. Details of the first experiment (experiment A) were described in Reid et al. (15). A maize hybrid (DeKalb DK415) was planted in a $4 \times 12$ factorial experiment arranged in a split-plot design with three blocks in Ottawa, Canada, in 1992, 1993, and 1994. Four inoculation treatments were randomized among the main plot units so each treatment consisted of 12 single-row subplot units that corresponded to 12 harvest times. Inoculation treatments consisted of $F$. graminearum, $F$. verticillioides, the two species mixed together, and a control treatment of sterile water. After inoculation, plots were irrigated with 2 to $5 \mathrm{~mm}$ of water daily in the late afternoon for 28 days. Ears were collected at 12 harvest times: 2, 4, 6, 8, 10, 12, 14, 18, 22, 35, 49, and 63 days after inoculation. In 1992 and 1993, an additional five harvest times were added (1, 3, 5, 20, and 26 days after inoculation). Harvested ears were bulked within each row and hand shelled. Each sample was mixed thoroughly and a subsample was freeze-dried and ground to a fine powder for analyses of deoxynivalenol (DON) and fumonisin $\left(\mathrm{FB}_{1}\right)$.

Ear samples were rated for disease severity using a 7-class rating system where $1=$ no visual infection, $2=1$ to $3 \%, 3=4$ to $10 \%, 4=11$ to $25 \%, 5=26$ to $50 \%, 6=51$ to $75 \%$, and $7=>75 \%$ of the kernels exhibiting visible disease symptoms (14). This scale represents a relatively quick and convenient method for quantifying disease severity but is logarithmic in nature. It can be converted to percent visual infection $(\% G)$, which is more closely related to the amount of Fusarium growth per ear. This conversion used a polynomial relating percent infection to the rating scale. Scale values of $1,2,3$, and 4 , etc., correspond to $G$ values of $0,1.5,7$, and $18 \%$, etc., which were the middle value of percent visible infection of each class. Average hourly values of temperature and relative humidity and daily precipitation were recorded at the experimental site. Air temperatures were measured throughout the experiment, and silk channel temperatures were measured for about the first 20 days until the silk channel no longer existed due to cob growth. Silk channel temperatures were used in the model when available and then air temperatures were used. These silk channel temperatures were highly correlated with and quite similar to air temperatures (15). Substituting air temperatures for silk channel temperatures had virtually no impact on model calculations.

In a second experiment (experiment $B$ ), nine inoculation times $(0,1,3,6,9,12,15,20$, and 30 days after silk emergence) were randomly assigned to three blocks of nine four-row plots of the maize hybrid used in experiment A. Three inoculation treatments ( $F$. graminearum, $F$. verticillioides, and a sterile water control) were randomized among the four subplot rows. The inoculation procedures were the same as used in experiment A. Each subplot row consisted of 10 plants in a $3.8 \mathrm{~m}$ long row with $76 \mathrm{~cm}$ between rows. Irrigation procedures were repeated as in experiment $\mathrm{A}$, and air temperatures and precipitation were recorded as in experiment A. At normal grain harvest in the middle of October, 10 ears (one from each plant) were hand-picked, husked, and evaluated for ear rot symptoms using the 7-class rating system described in experiment $A$.

In experiment $\mathrm{A}$, we inoculated 5 days after first silk appearance and measured percent visual infection at specified time intervals during the growth period. In experiment B, we inoculated at specified times after first silk appearance and measured percent visual infection at harvest time.

Parameterization. There are a number of empirical coefficients in this model. The $a$ coefficients $\left(a_{1}\right.$ to $\left.a_{5}\right)$ had well-defined values when sums of squares between model calculations and ob- 
servations from experiment A were plotted as functions of each coefficient and could be solved for by fitting the model to percent visual infection using a nonlinear least squares fitting algorithm (7) with numerical differentiation. In total, 138 observations over the 3 years of experiment A were used to solve for these coefficients. There are six coefficients because $a_{3}$ was species-dependent and therefore had two values. The $c$ coefficients did not have well-defined minima, were interdependent on other coefficients, and could not be solved directly. These coefficients were determined in a sensitivity study in which values were varied systematically to obtain optimum fits (5). For example, $c_{1}$ was dependent to some extent on $a_{1}$. Values of $c_{2}$ and $c_{3}$ determined the amounts of interference in the silks and kernels, respectively, but model output was insensitive to where the interference took place. Therefore, we assumed approximately equal interference in the silks and kernels. Initial values were also set by sensitivity studies since, to some extent, they interacted with the $a_{1}$ coefficient. Values of 0.3 and $0.6 \%$ visual infection were set for $G_{0}$ and $G_{E}$ when species were inoculated separately, respectively. When the inoculum was mixed, values of $G_{0}$ were set at 0.15 for each species and the total amount of infection was calculated separately for each species and then added together.

We intended to use the model, as described above, to calculate values of infection for the conditions of experiment $B$ as an independent test. That is, we started the model at each inoculation time in experiment B, ran it over the growing period, and compared the calculated percent visual infection with that measured at harvest. However, preliminary results showed the need for a silk function to account for the effect of silk age on growth. Reid et al. (13) showed that disease ratings increased to a maximum value and then decreased as silk inoculation times increased from time of first silk appearance. They made no attempt to separate age factors from environmental affects. In this study, we developed a silk function $\left(S_{F}\right)$ to account for silk aging using the data from experiment B. $S_{F}$ was developed as a function of modified growing degree days (MGDD) (18) accumulated from first silk appearance. $S_{F}$ was set at a small negative number $\left(d_{1}\right)$ at first silk appearance, increased to one at a specified number of MGDD $\left(d_{2}\right)$, remained at one until another specified number of MGDD $\left(d_{3}\right)$, where it declined with a slope $d_{4}\left(\mathrm{MGDD}^{-1}\right)$ to a lower limit of $d_{5}$. These coefficients ( $d_{1}$ to $d_{5}$ ) were determined by comparing model calculations to the disease infection levels from experiment B through a sensitivity analysis with the exception of $d_{4}$, which was solved directly by the nonlinear least squares method used previously but with the data from experiment B. The coefficients $b_{1}$ and $b_{2}$ from equation 13 were solved by least squares using data from Reid et al. (16).

\section{RESULTS AND DISCUSSION}

Infection from natural sources of inoculum, as determined from the control treatment, were negligible so that using percent visual infection for determining coefficients was valid. Values of all coefficients are shown in Table 1 . Note, only the $a$ coefficients and $d_{4}$ have standard errors that were calculated by the algorithm (7). The temperature function for each species (equation 2) was compared with growth rates of Fusarium spp. grown on infected silks on agar plates under controlled conditions from Reid et al. (15) (Fig. 1). We set values of $T_{\mathrm{opt}}$ and $T_{\max }$ for $F$. graminearum based on this data. However, the equivalent values for $F$. verticillioides were estimates assuming $F$. verticillioides grew faster at higher temperatures. A frequency distribution of temperatures for the first 40 days after inoculation (Fig. 2) indicated that a large percentage of the temperatures were below $30^{\circ} \mathrm{C}$. As well, conditions in Ottawa, ON were not conducive to growth of $F$. verticillioides because growth rates of this fungus were relatively small each year. Therefore, the fitting procedure was not able to establish values for $T_{\mathrm{opt}}$ and $T_{\max }$ for $F$. verticillioides.
The data in Figure 1 suggests that $T_{\min }$ would be in the range of 22 to $24^{\circ} \mathrm{C}$. However, as stated above, other studies $(1,6)$ have indicated that growth of $F$. verticillioides can occur below $22^{\circ} \mathrm{C}$. $F$. graminearum causes head blight in wheat (19), which happens during the relatively cool temperatures of June in Ontario. Therefore, a lower temperature limit $\left(T_{\min }\right)$ of $15^{\circ} \mathrm{C}$ seemed reasonable although research is continuing on temperature functions.

The $a_{2}$ coefficient was physically related to the boundary layer resistance of Rao et al. (12), which is related to the dimensions of the ear and the wind velocity in the vicinity of the ear. In this case, $a_{2}$ was also related to the water conductivity of the husks since part of the film of water was in the husks and between kernels and husks. Wind velocity at ear level was not measured in this experiment and is seldom available for field experiments. As well, there is a dearth of information as to how water moves back and forth from the husk surface to the kernels where growth of these fungi takes place. Thus, as a first approximation, $a_{2}$ was assumed to be a
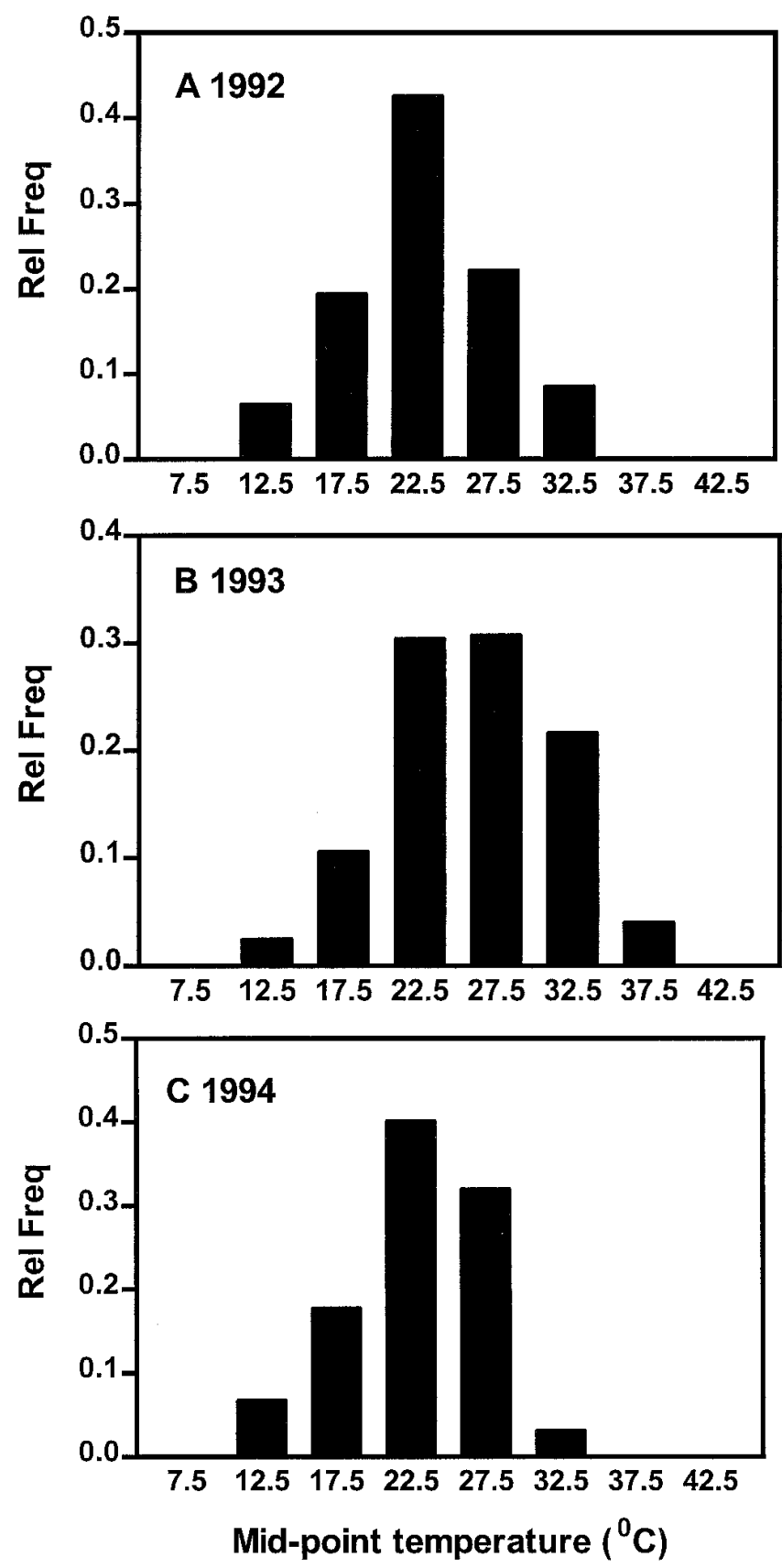

Fig. 2. Relative frequency of temperatures for the first 40 days after inoculation for the conditions of experiment A. 
simple constant that was solved for by the nonlinear algorithm and represented a value for an average-sized ear for average wind conditions. Even though the data was used to solve for this coefficient, $a_{2}$ will be related to the above factors that influence evaporation but not to the nature of how the water was applied. That is, we would still expect the value of $a_{2}$ in Table 1 to have the same value whether or not overhead irrigation was used to supplement rainfall unless irrigation wets the ears differently than rainfall. This is important for future use of this model for predicting occurrences of disease. The exact thickness of the film (equation 4) of water $(1 \mathrm{~mm})$ was not critical to the model. An increase or decrease of up to $50 \%$ (determined by sensitivity studies using the data from experiment A) could be tolerated because $a_{2}$ and $a_{3}$ would adjust to these changes.

The $a_{3}$ coefficients represented the response of Fusarium spp. to the wetness of the leaf, which was, in turn, determined by precipitation and the evaporative conditions of the atmosphere. The $a_{3}$ coefficient for $F$. graminearum was almost twice the value for $F$. verticillioides (Table 1). The temperature response functions for the temperatures measured in this experiment were very similar. Therefore, the large difference between the growth of the two species was determined mainly by $a_{3}$.

TABLE 2. Time from silk inoculation to when the infection reaches the ear for Fusarium graminearum (Gr), F. verticillioides (Ve), and for mixed inoculation $(\mathrm{Gr}+\mathrm{Ve})$, as calculated from the model described in the text, and the initial values $\left(G_{E}\right)$ of percent visual infection of the ear used in the model

\begin{tabular}{lccl}
\hline Year & Species & Time $(d)$ & \multicolumn{1}{c}{$G_{E}$} \\
\hline 1992 & $\mathrm{Gr}$ & 13.54 & 0.6 \\
& $\mathrm{Ve}$ & 15.63 & 0.6 \\
& $(\mathrm{Gr}+\mathrm{Ve})$ & 13.79 & $0.27(\mathrm{Gr})$ \\
& & & $0.33(\mathrm{Ve})$ \\
1993 & $\mathrm{Gr}$ & 15.46 & 0.6 \\
& $\mathrm{Ve}$ & 21.54 & 0.6 \\
& $(\mathrm{Gr}+\mathrm{Ve})$ & 16.42 & $0.25(\mathrm{Gr})$ \\
& & & $0.35(\mathrm{Ve})$ \\
1994 & $\mathrm{Gr}$ & 9.67 & 0.6 \\
& $\mathrm{Ve}$ & 12.75 & 0.6 \\
& $(\mathrm{Gr}+\mathrm{Ve})$ & 10.71 & $0.25(\mathrm{Gr})$ \\
& & & $0.35(\mathrm{Ve})$ \\
\hline
\end{tabular}

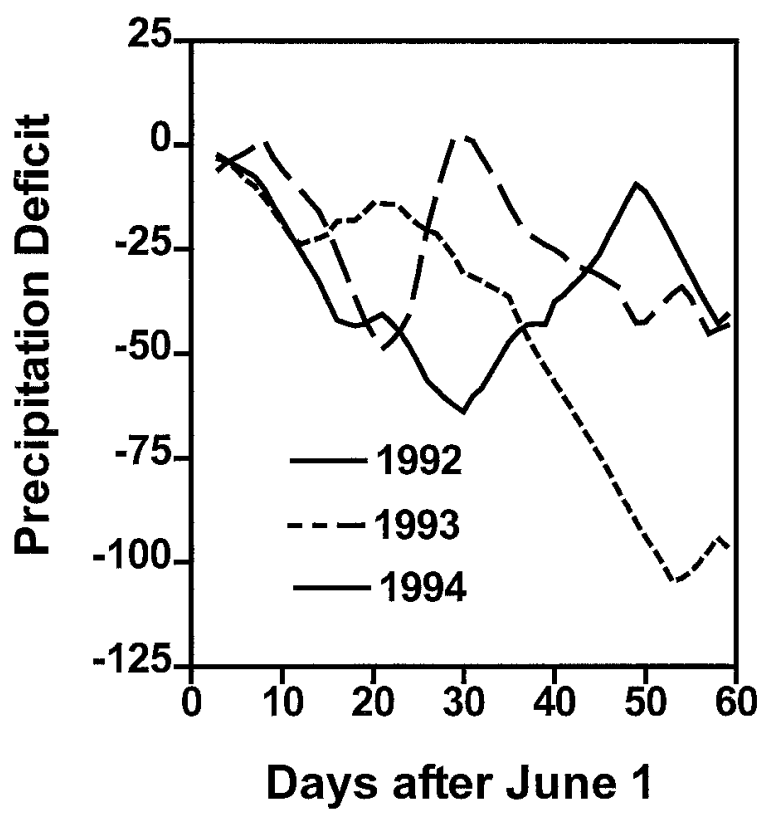

Fig. 3. Five-day moving averages of cumulative precipitation deficits (millimeters) for 60 days after 1 June for the 3 years (1992 to 1994) of experiment A.
Initial starting values and growth in silks are very important and are interdependent on growth coefficients. We chose an initial value $\left(G_{0}\right)$ of $0.3 \%$ to leave room for natural sources of disease invasion when we would assume a value between 0 and $0.3 \%$. A $G_{E}$ value of $0.6 \%$ was dependent on $c_{1}$ and $a_{1}$. We determined all this by sensitivity studies using data from experiment $\mathrm{A}$ and determining reasonable values for the time it took $G$ to increase from 0.3 to $0.6 \%$. These times and component values of $G_{E}$ are shown in Table 2. The component values represented the amounts of growth in the silks of each species for the mixed inoculum treatment. There was a possibility that amounts of inoculum reached saturated levels very quickly and that actual amounts of inoculum above the levels used in the mixed inoculum treatment would not affect results. If this was the case, this saturating effect can be programmed into the model but it would also be necessary to increase the values of the interference coefficients in the model.

The $a_{5}$ coefficient is intriguing. It had a value of 0.192 in 1993 (Table 1). It was assumed to be 1.0 in the other years. During 1993, growth rates down the silks were less than one-third that of the other years if all other factors were the same. Reid et al. (15) showed substantially less ergosterol in the silks for this year. We assumed that this was due to preinoculation weather conditions. Precipitation deficits during June and July indicated much drier conditions in 1993 compared with 1992 and 1994 (Fig. 3), which made the silks less susceptible to infection. We quantified this by calculating the precipitation deficit over the 40 days before the day of inoculation and relating it to this growth coefficient, which was 1.0 in 1992 and 1994 and 0.192 in 1993 (Fig. 4). The nonlinear nature of the function in Figure 4 was such that for most years this coefficient would equal 1.0 and not be a factor in the model, which was a reasonable assumption that has not been verified. Note that the function in Figure 4 was not used in the analyses in this paper and does not affect the results. Only the data points were used. The function was derived for future comparisons with other data sets and could change with more analyses.

The Fusarium spp. growth model compared quite well to the disease rating data and followed the yearly patterns of Fusarium spp. growth reasonably well both for isolated species and when they were inoculated together (Fig. 5). Note that the model follows a general sigmoid pattern that after inoculation there was a period with slow progress of fungal growth followed by exponential growth then by another slow growth. The overall $R^{2}$ was 0.91 with a root mean square error of standard error of estimate (SEE) of $2.42 \%$, although this was not an independent test of the model. It is obvious from Figure 5 that in isolation, $F$. graminearum grew at a higher rate than $F$. verticillioides. However, when the two species were mixed together, values of percent visual infection for Fusarium spp. were much less than

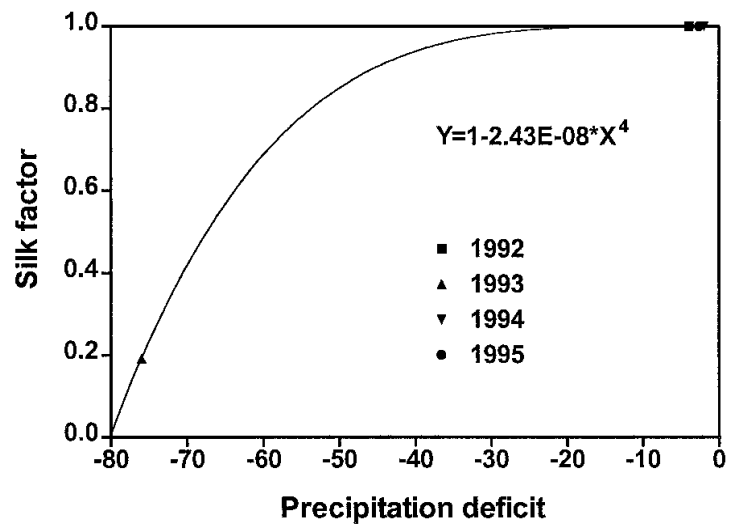

Fig. 4. Water stress growth coefficients $\left(a_{5}\right)$ for silks as a function of precipitation deficits ( $x$ axis) (millitmeters) summed over 40 days prior to silking for 1992, 1993, 1994, and 1995. 
when $F$. graminearum was inoculated alone. Part of this is because only half the amount of inoculum was used for each species to prepare the mixed inoculum treatment. This is accounted for in the model when we divided $G_{0}$ by two. However, we also had to assume some interference by $F$. verticillioides on the growth of $F$. graminearum to optimize the fits.

Simulated growth of Fusarium on the ears was exponential (equation 1), which enabled the model to calculate very large growth rates under favorable conditions. This growth was controlled in the latter parts of the growing period by lower temperatures because at these later stages of growth water availability was not as variable as temperatures (data not shown). Thus, the value of $T_{\min }$ was a critical factor in the model. We found that lowering $T_{\text {min }}$ decreased the growth coefficient $\left(a_{1}\right)$ and made growth more
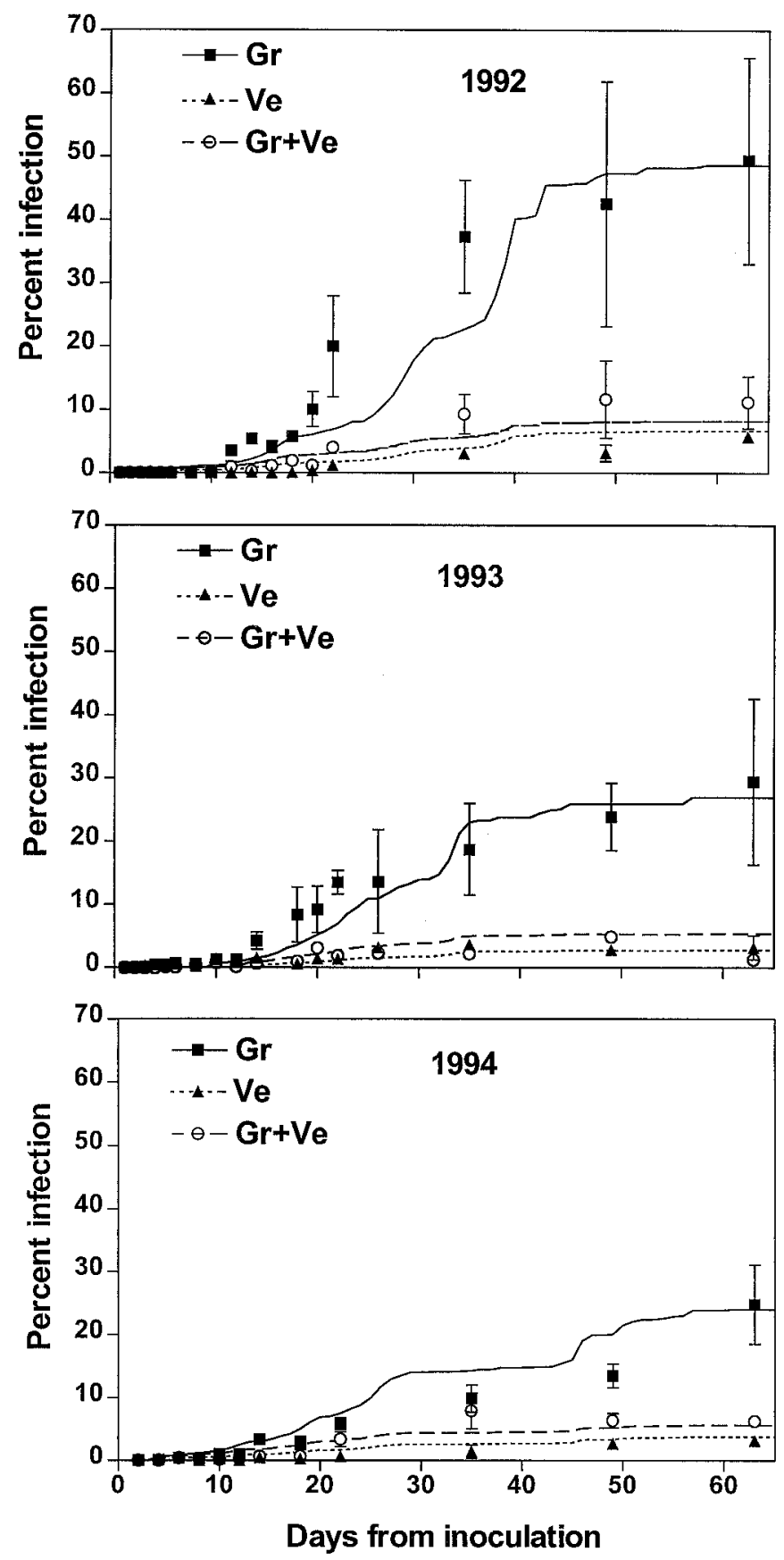

Fig. 5. Percent visual infection of maize ears as related to days from inoculation for Fusarium graminearum (Gr) by itself, F. verticillioides (Ve) by itself, and for the two species inoculated together $(\mathrm{Gr}+\mathrm{Ve})$ over 3 years. Lines indicate model calculations. Inoculation took place 5 days after silk emergence. Error bars represent the standard error of the means. linear with time. Values of $T_{\min }$ lower than $15^{\circ} \mathrm{C}$ resulted in relatively large SEEs with the data of Figure 5 . Therefore, setting $T_{\min }$ to $15^{\circ} \mathrm{C}$ was a combination of using a value that worked well in the model but also seemed the most reasonable based on what we know of the Fusarium growth from the literature.
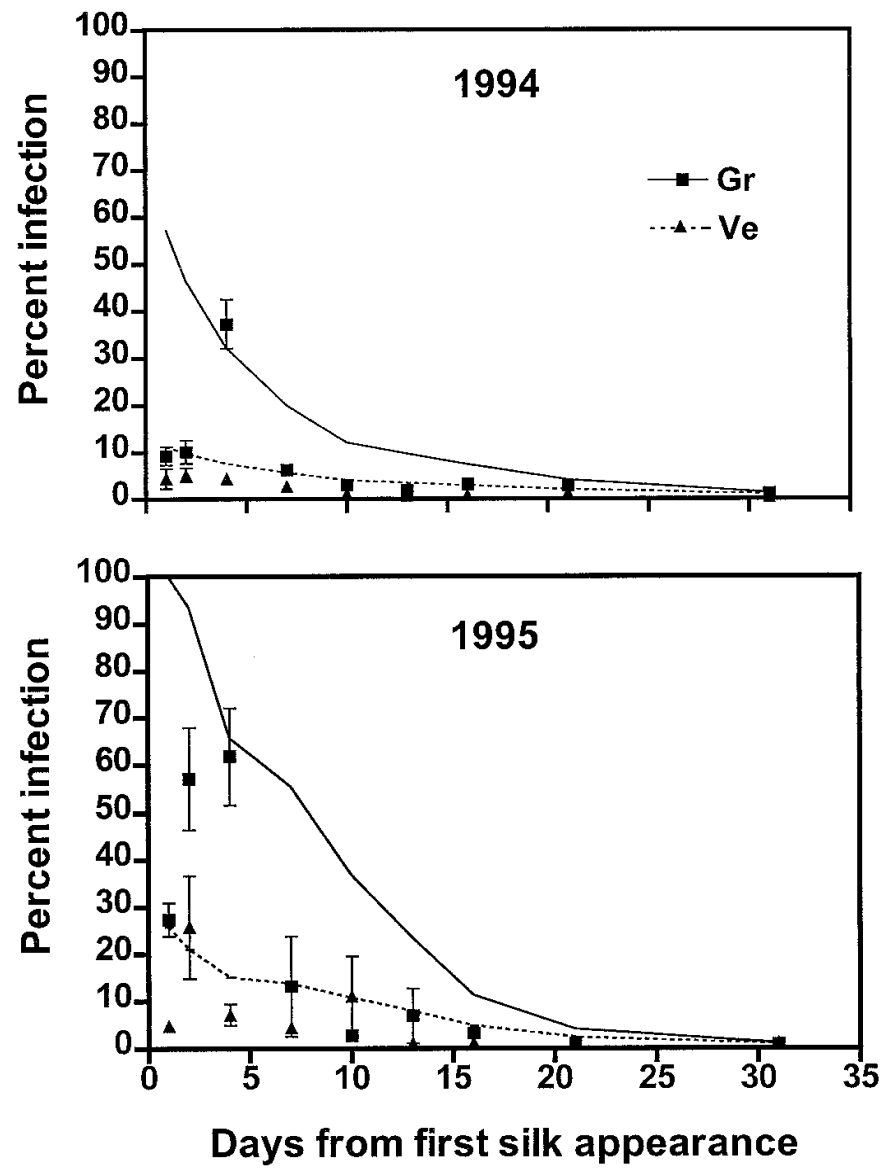

Fig. 6. Percent visual infection as related to time of inoculation (days from first silk appearance) for Fusarium graminearum $(\mathrm{Gr})$ and $F$. verticillioides (Ve) with no silk function $\left(F_{S}=1\right)$. Symbols represent percent visual infection measured at harvest time. Lines are corresponding model calculations. Error bars represent the standard error of the means.

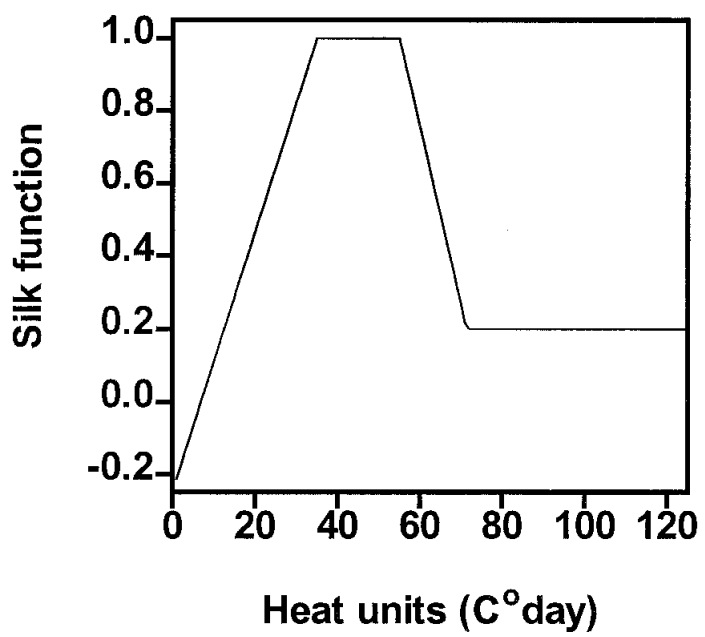

Fig. 7. The silk function $\left(F_{S}\right)$ related to modified growth degree days accumulated from the time of first silk appearance as used for the conditions of experiment $\mathrm{B}$. 
The silk function was another key factor. When $S_{F}$ was set to one throughout the growth periods, serious discrepancies appeared between model calculations and measurements of experiment $\mathrm{B}$ (Fig. 6), particularly 1 day after silk emergence. When we applied the silk function (Fig. 7), the model calculations were much closer to the observations of experiment $\mathrm{B}$ (Fig. 8). The $R^{2}$ improved from 0.58 to 0.89 and the SEE decreased from 20.8 to $5.9 \%$ although this was not an independent test of the model because the data of experiment B was used to develop the silk function. When model calculations were repeated for conditions of experiment A with the silk function in place, they did not change from the original model calculations for experiment A. However, a key assumption was that the silks aged from the outside in and that infection grew down the silk tubules faster than the aging process. This assumption was necessary because it took 13 to 22 days before there were significant levels of infection on the ears (Fig. 5; Table 2) and we assumed that this lag was due to the time taken for the Fusarium to grow down the silks. This may not be true. Fusarium may grow down silk more rapidly than what was assumed here but took time to switch from silk growth to growth on kernels. More research is required to resolve this aspect of Fusarium growth. What the model showed was that the condition of silks played a major role in disease infection. There appeared to be a very narrow window (4 to 6 days after silk emergence) for optimum conditions for infection. When silks first emerged they seemed to resist disease invasion but quickly ( 2 to 4 days) became susceptible. Maximum susceptibility $\left(S_{F}=1\right)$ only lasted for several more days when susceptibility decreased to a plateau level. This plateau level was put into the function so that there
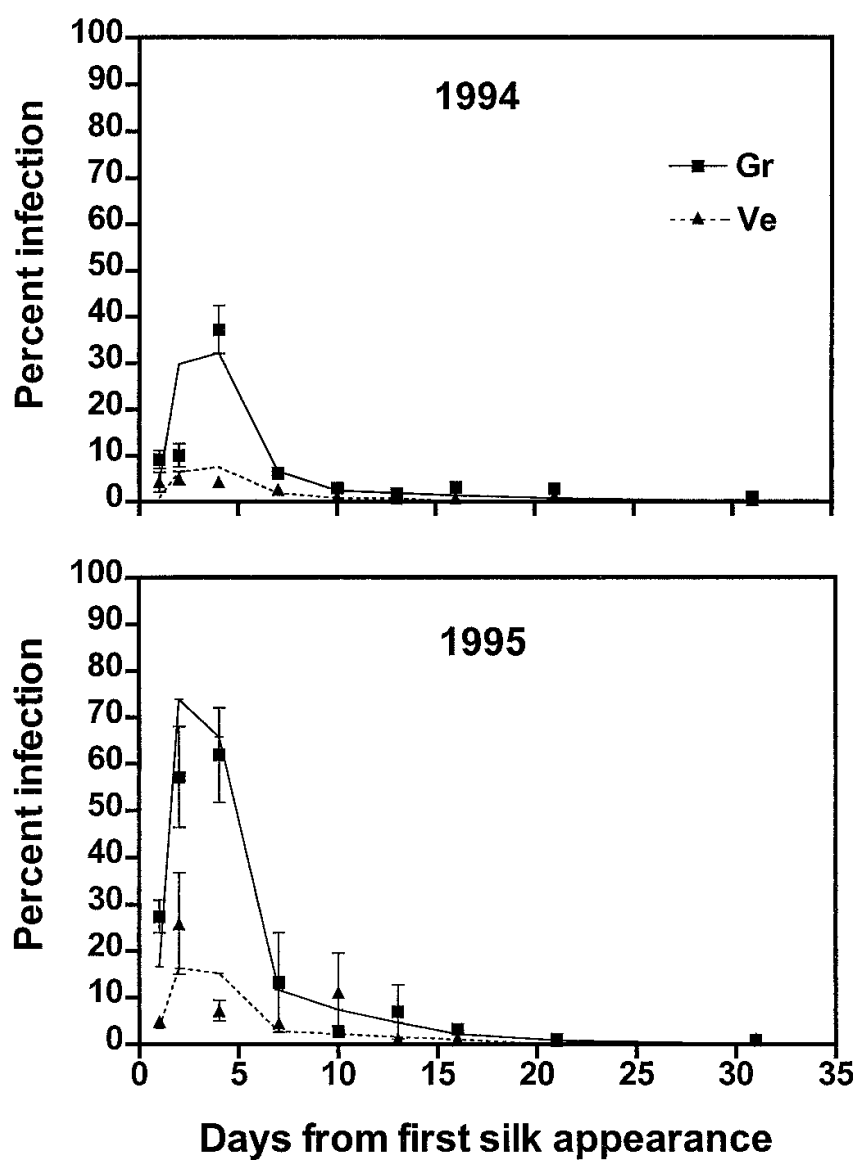

Fig. 8. Percent visual infection as related to time of inoculation (days from first silk appearance) for Fusarium graminearum $(\mathrm{Gr})$ and $F$. verticillioides (Ve) using the silk function depicted in Figure 7. Symbols represent percent visual infection measured at harvest time. Lines are corresponding model calculations. Error bars represent the standard error of the means. would be some risk, however small, of infection through the silks during the latter stages of the growth period. This plateau level of 0.20 instead of zero improved the fit only marginally but it could be important for other data sets. Reid et al. (13) showed some disease in maize ears at harvest time when silks were inoculated 10 to 16 days from first silk appearance for two other hybrids.

Equation 13 related percent visual infection to toxin levels from Reid et al. (16) well $\left(R^{2}=0.87\right)$ (Fig. 9). However, Reid et al. (15) showed that sometimes very high levels of DON can occur at relatively low levels of percent visual infection (Fig. 9). The fit in Figure 9 is conservative since two outlying points (which appeared to be spurious) were not plotted. Therefore, the relationship (equation 13) presented here may not be adequate. Further work is being conducted similar to Pitt (10) in which toxin formation is related to rate of growth rather than the amount of growth. The low levels of $\mathrm{FB}_{1}$ corresponded to the small amount of $F$. verticillioides calculated by the model.

The model is developed according to most conventional thinking that $F$. graminearum grows better in wetter, cooler conditions than $F$. verticillioides. Under the weather conditions of Ontario, both $F$. graminearum and $F$. verticillioides are relatively common. However, in some years (usually wet years), F. graminearum is the predominant species that produces high levels of toxins. Results from this study suggest that $F$. graminearum has to outgrow the $F$. verticillioides sufficiently to overcome the interference. In hotter, drier conditions, F. verticillioides would prob-
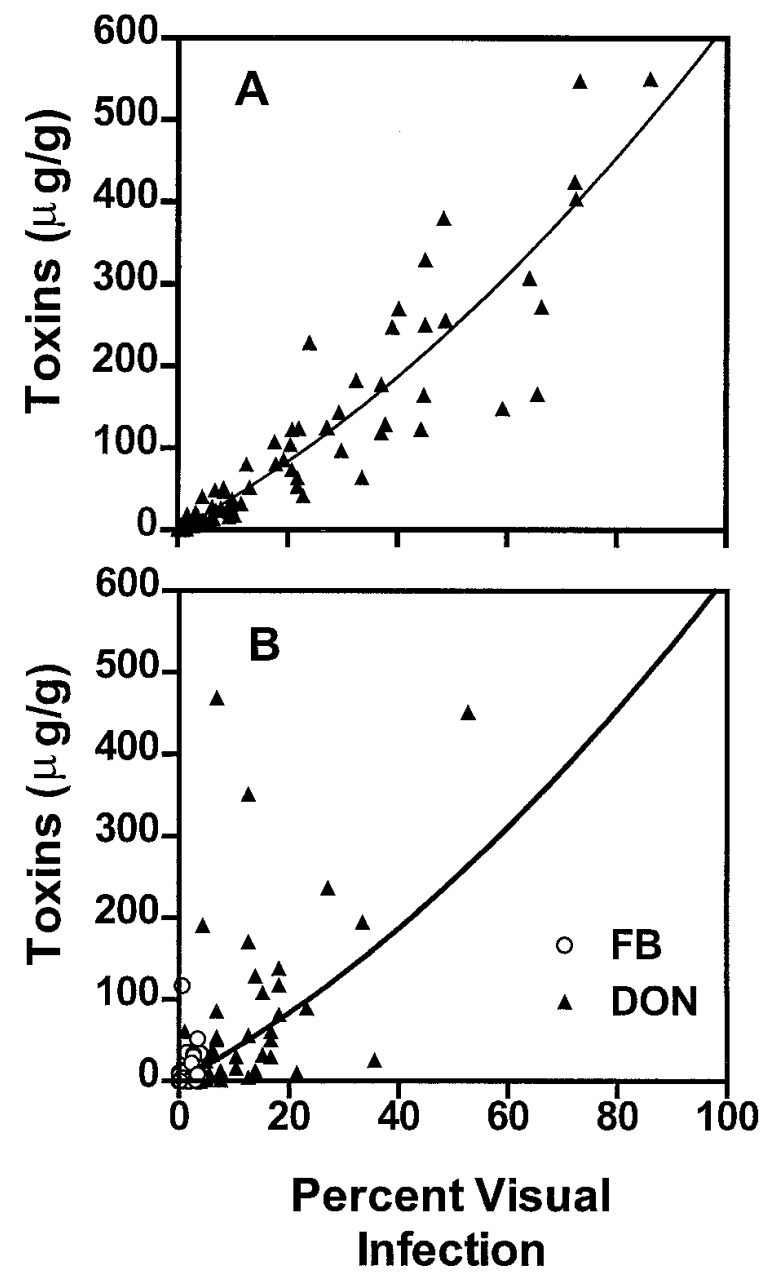

Fig. 9. A, Toxin concentrations $(y)$ are related to percent visual infection $(x)$ using the data from Reid et al. (16). The solid line $\left(y=3.66 x+0.025 x^{2}\right)$ (equation 13) is a least squares fit to the data with $R^{2}$ of 0.85 . B, The data from Reid et al. (15) is compared with the same function. DON = deoxynivalenol; $\mathrm{FB}=$ fumonisin. 
ably predominate since it seems to have the ability to interfere with the growth of $F$. graminearum.

The model development in this study was only a first step in a conceptual or mechanistic model of Fusarium growth. Because we used the data in experiment B to develop a silk function, we did not have a truly independent test of the model. However, model calculations for the conditions of experiment B when $S_{F}$ equaled one (those made on the fourth day after first silk appearance) were in fact independent. This meant that only four measurements were available for testing and it is fair to ask how the model calculations would compare with other independent data. In this case, a truly independent data set will be difficult to obtain because some of the coefficients will change with hybrid. The silk function may be in this category. However, the silk function was not just a method of making the model fit the data. It quantified changes in the susceptibility of silks to invasion of Fusarium with time and temperature and has inherent usefulness on its own. The growth coefficients $a_{1}$ and $a_{3}$ will almost certainly change with susceptibility of hybrids but may be a useful way of quantifying susceptibility to Fusarium disease. Therefore, analyzing more data with this model may be aimed at determining how various coefficients change with hybrid more than at independently testing the model.

The environment of Ontario is not conducive to growth of $F$. verticillioides. It would be unreasonable to assume that we adequately tested a model for this species for all locations. In Ontario, $F$. verticillioides does not produce enough toxins to be a problem. F. graminearum is the species of most concern. The model, as presented, only dealt with infection through silks. Other means of the fungi for infecting the kernels, such as through insect wounds or bird damage, has not been considered here but are topics for ongoing research.

The ultimate use of this model is to calculate geographical locations and years when toxins are produced in large enough quantities of concern. However, we are several steps away from this. For one, the toxin submodel needs to be improved. As well, we have not included any aspects of natural sources of inoculum. However, the model does have uses in its present form. For example, the model quantified the importance of silk age to successful infection of maize ears by two species of Fusarium and demonstrated the importance of inoculating silks 4 to 6 days after silk emergence for testing silk resistance to infection of maize lines. If all lines do not silk within several days, they have to be inoculated on different days, which would result in having different environmental conditions for Fusarium infection and confounds inherent resistance with environmental conditions. This could be avoided if the percent visual infection measured on each line is expressed as a fraction of the percent visual infection calculated by this model.

In summary, a mathematical model was developed that successfully simulates growth of $F$. graminearum and $F$. verticillioides under separate and mixed silk inoculations. It is the first stage of a model to calculate natural levels of maize ear infection of Fusarium spp. for field conditions.

\section{ACKNOWLEDGMENTS}

We thank L. Ranacher and B. Wilson for technical assistance.

\section{LITERATURE CITED}

1. Alberts, J. F., Gelderblom, W. C. A., Thiel, P. G., Marasa, W. F. O., Van Schalhwyk, D. J., and Behrend, Y. 1990. Effect of temperature and incubation period on production of Fumonisin $\mathrm{B}_{1}$ by Fusarium moniliforme. Appl. Environ. Microbiol. 56:1729-1733.

2. Baier, W. 1971. Evaluation of latent evaporation estimates and their conversion to potential evaporation. Can. J. Plant Sci. 51:255-266.

3. Baier, W., and Robertson, G. W. 1965. Estimation of latent evaporation from simple weather observations. Can. J. Plant Sci. 45:276-284.

4. Campbell, C. L., and Madden, L. V. 1990. Introduction to Plant Disease Epidemiology. John Wiley \& Sons, New York.

5. Hunt, L. A., Pararajasingham, S., Jones, J. W., Hoogenboom, G., Imamura, D. T., and Oghoshi, R. M. 1993. GENCALC-Software to facilitate the use of crop models for analysing field experiments. Agron. J. 85:1090-1094.

6. Marin, S., Magan, N., Serra, J., Ramos, A. J., Canela, R., and Sanchis, V. 1999. Fumonisin $\mathrm{B}_{1}$ production and growth of Fusarium moniliforme and Fusarium proliferatum on maize, wheat, and barley grain. J. Food Sci. 64:921-924.

7. Marquardt, D. W. 1963. An algorithm for least squares estimation of non-linear parameters. J. Soc. Ind. Appl. Math. 11:431-441.

8. Miller, J. D., Savard, M. E., Schaafsma, A. W., Siefert, K. A., and Reid, L. M. 1995. Mycotoxins production by Fusarium moniliforme and Fusarium proliferatum from Ontario and presence of fumonisins in the 1993 corn crop. Can. J. Plant Pathol. 17:233-239.

9. Moschini, R. C., and Fortugno, C. 1996. Predicting wheat head blight incidence using models based on meteorological factors in Pergamino, Argentina. Eur. J. Plant Pathol. 102:211-218.

10. Pitt, R. E. 1993. A descriptive model of mold growth and aflatoxin formation as affected by environmental conditions. J. Food Prod. 56:139146.

11. Prelusky, D. B., Rotter, B. A., and Rotter, R. G. 1994. Toxicology of mycotoxins. Pages 359-403 in: Mycotoxins in Grain: Compounds Other than Aflatoxin. J. D. Miller and H. L. Trenholm, eds. Eagan Press, St. Paul, MN.

12. Rao, P. S., Gillespie, T. J., and Schaafsma, A. W. 1998. Estimating wetness duration on maize leaves from meteorological observations. Can. J. Soil Sci. 78:149-154.

13. Reid, L. M., Bolton, A. T., Hamilton, R. I., Woldemariam, T., and Mather, D. E. 1992. Effect of silk age on resistance of maize to Fusarium graminearum. Can. J. Plant Pathol.14:293-298.

14. Reid, L. M., Hamilton, R. I., and Mather, D. E. 1996. Screening maize for resistance to Gibberella ear rot. Agric. Agri-Food Can. Tech. Bull. Publ. 196-5E

15. Reid, L. M., Nicol, R. W., Ouellet, T., Savard, M., Miller, J. D., Young, J. C., Stewart, D. W., and Schaafsma, A. W. 1999. Interaction of Fusarium graminearum and $F$. moniliforme in maize ears: Disease progress, fungal biomass, and mycotoxin accumulation. Phytopathology 89:10281037.

16. Reid, L. M., Stewart, D. W., and Hamilton, R. I. 1996. A 4-year study of the association between Gibberella ear rot severity and deoxynivalenol concentration. J. Phytopathol. 144:431-436.

17. Shurtleff, M. C., ed. 1984. Compendium of Corn Diseases. The American Phytopathological Society, St. Paul, MN.

18. Stewart, D. W., Dwyer, L. M., and Carrigan, L. 1998. Phenological temperature response of maize. Agron. J. 90:73-79.

19. Sutton, J. C. 1982. Epidemiology of wheat head blight and maize ear rot caused by Fusarium graminearum. Can. J. Plant Pathol. 4:195-209.

20. Sutton, J. C., Baliko, W., and Liu, H. J. 1980. Fungal colonization and zearalenone accumulation in maize ears injured by birds. Can. J. Plant Sci. 60:453-461.

21. Vigier, B., Reid, L. M., Seifert, K. A., Stewart, D. W., and Hamilton, R. I. 1997. Distribution and prediction of Fusarium species associated with maize ear rot in Ontario. Can. J. Plant Pathol. 19:60-65.

22. Waggoner, P. E., Horsfall, J. G., and Lukens, R. J. 1972. EPIMAY, a simulator of southern corn leaf blight. Conn. Agric. Exp. Stn. Bull. 729. 